\title{
Mannitol versus hypertonic saline for brain relaxation in patients undergoing craniotomy - A Cochrane Systematic Review
}

\author{
Hemanshu Prabhakar, Gyaninder P. Singh, Mani Kalaivani' , Vidhu Anand ${ }^{2}$
}

\begin{abstract}
Background: Patients with brain tumour usually suffer from increased pressure in the skull due to swelling of brain tissue. A swollen brain renders surgical removal of the brain tumour difficult. To ease surgical tumour removal, measures are taken to reduce brain swelling, often referred to as brain relaxation. Brain relaxation can be achieved with intravenous fluids such as mannitol or hypertonic saline. The objective of this review was to compare the effects of mannitol versus those of hypertonic saline on intraoperative brain relaxation in patients undergoing craniotomy. Methods: We searched the Cochrane Central Register of Controlled Trials (2013, Issue 10), MEDLINE through OvidSP (I966 to October 2013) and EMBASE through OvidSP (1980 to October 20I3). We also searched specific websites, such as www.indmed.nic.in, www.cochrane-sadcct.org and www.clinicaltrials.gov. We included randomised controlled trials (RCTs) that compared the use of hypertonic saline versus mannitol for brain relaxation. We also included studies in which any other method used for intraoperative brain relaxation was compared with mannitol or hypertonic saline. The primary outcomes were longest follow-up mortality, Glasgow outcome scale score at 3 months and any adverse event related to mannitol or hypertonic saline. The secondary outcomes were intraoperative brain relaxation, Intensive Care Unit (ICU) stay, hospital stay and quality of life. We used standardised methods for conducting a systematic review as described by the Cochrane Handbook for Systematic Reviews of Interventions. Two review authors independently extracted details of trial methodology and outcome data from reports of all trials considered eligible for inclusion. All analyses were made on an intention-to-treat basis. We used a fixed-effect model when no evidence was found of significant heterogeneity between studies and a random-effects model when heterogeneity was likely. Results: We included six RCTs with 527 participants. Only one RCT was judged to be at low risk of bias. The remaining five RCTs were at unclear or high risk of bias. No trial mentioned the primary outcomes of longest follow-up mortality, Glasgow outcome scale score at 3 months or any adverse event related to mannitol or hypertonic saline. Three trials mentioned that the secondary

Departments of Neuroanaesthesiology and Critical Care and ${ }^{1}$ Biostatistics, All India Institute of Medical Sciences, New Delhi, India, ${ }^{2}$ Department of Medicine, University of Minnesota, Minneapolis, MN, USA

\section{Address for correspondence:}

Dr. Hemanshu Prabhakar, Department of Neuroanaesthesiology and Critical Care, All India Institute of Medical Sciences, Ansari Nagar, outcomes of intraoperative brain relaxation, hospital stay, ICU stay and quality of life were not reported in any of the trials. Brain relaxation was inadequate in 42 of 197 participants in the hypertonic saline group and 68 of 190 participants in the mannitol group. The risk ratio for brain bulge or tense brain in the hypertonic saline group was 0.60 (95\% confidence interval $0.44-0.83$, low-quality evidence). One trial reported ICU and hospital stay. The
\end{abstract} New Delhi - 110 029, India.

E-mail: prabhakaraiims@yahoo.co.in

\begin{tabular}{|l|l|}
\hline \multicolumn{2}{|c|}{ Access this article online } \\
\hline Quick Response Code: & Website: \\
\hline & www.jnaccjournal.org \\
\cline { 2 - 2 } & \\
\hline & DOI: \\
\hline
\end{tabular}

This is an open access article distributed under the terms of the Creative Commons Attribution-NonCommercial-ShareAlike 3.0 License, which allows others to remix, tweak, and build upon the work non-commercially, as long as the author is credited and the new creations are licensed under the identical terms.

For reprints contact: reprints@medknow.com

How to cite this article: Prabhakar H, Singh GP, Kalaivani M, Anand $\mathrm{V}$. Mannitol versus hypertonic saline for brain relaxation in patients undergoing craniotomy - A Cochrane Systematic Review. J Neuroanaesthesiol Crit Care 2017;4:99-107.

This article is based on a Cochrane Review published in the Cochrane Database of Systematic Reviews (CDSR) 2014, Issue 7, DOI: 10.1002/14651858.CD010026 (see www.thecochranelibrary.com for information). Cochrane Reviews are regularly updated as new evidence emerges and in response to feedback, and the CDSR should be consulted for. the most recent version of the review 
mean (standard deviation [SD]) duration of ICU stay in the mannitol and hypertonic saline groups was I.28 (0.5) and $1.25(0.5)$ days $(P-0.64)$, respectively; the mean (SD) duration of hospital stay in the mannitol and hypertonic saline groups was $5.7(0.7)$ and $5.7(0.8)$ days $(P-1.00)$, respectively. Conclusions: From the limited data available on the use of mannitol and hypertonic saline for brain relaxation during craniotomy, it is suggested that hypertonic saline significantly reduces the risk of tense brain during craniotomy. A single trial suggests that ICU stay and hospital stay are comparable with the use of mannitol or hypertonic saline. However, focus on other related important issues such as long-term mortality, long-term outcome, adverse events and quality of life is needed.

Key words: Brain relaxation, craniotomy, hypertonic saline, mannitol

\section{INTRODUCTION}

One of the important goals of anaesthetic management for patients undergoing craniotomy is to provide a relaxed brain on which the surgeon can operate. This allows easy surgical manipulation and causes less damage to normal brain tissue. This, in turn, results in less secondary injury to the brain, which improves the patient's neurological outcome. Raised intracranial pressure results in a tense brain during the intraoperative period. Administration of mannitol is generally considered to be a 'gold standard' for the treatment of raised intracranial pressure. Hypertonic saline is another intravenous fluid that has effects comparable with those of mannitol in terms of reduction in intracranial pressure. ${ }^{[1-3]}$ Earlier works on the use of hypertonic saline in neurosurgical patients have shown promising results. ${ }^{[4,5]}$

Raised intracranial pressure during the intraoperative period results in bulging of brain and poor surgical exposure. Various treatment methods have been used by anaesthetists to reduce this intraoperative brain bulge. These methods include hyperventilation (increasing respiratory rate); drainage of cerebrospinal fluid; use of intravenous anaesthetic agents such as propofol and thiopentone; facilitation of venous drainage by positioning of patients with head up and use of osmotic agents, such as mannitol and hypertonic saline. These manoeuvres facilitate relaxation of the brain and surgery as less retraction pressure is required to separate the lobes of the brain.

Mannitol is a six-carbon sugar with a molecular weight of 182; it is available as $20 \%$ and $25 \%$ solution. Mannitol is rapidly infused intravenously in doses of $0.25-1 \mathrm{~g} / \mathrm{kg}$. As it is hyperosmolar, that is, has greater osmolality than blood, mannitol facilitates the shift of water from the brain into the vasculature. Hypertonic saline is the hyperosmolar solution of normal saline, which is a sodium chloride solution. It is commonly available in concentrations of $3 \%, 5 \%, 7.5 \%$ and $23 \%$. Hypertonic saline provides the advantage of not crossing the blood-brain barrier; therefore, it remains in the intravascular compartment and does not enter into brain tissue. ${ }^{[6]}$ Hypertonic saline has less of diuretic effect when compared with mannitol and thus maintains better cerebral perfusion pressure. ${ }^{[6]}$ Osmotic diuretics such as mannitol and hypertonic saline increase the osmolality of the blood, which shifts water from the brain to the intravascular compartment, that is, into the blood. Intravenous administration of hypertonic saline has been shown to improve cerebral perfusion. At the same time, brain oedema is reduced by the intervention, thus increasing compliance and decreasing intracerebral pressure. Hyperosmolar solutions such as mannitol and hypertonic saline have been used routinely to achieve brain relaxation in neurosurgical patients undergoing craniotomy. Both agents offer advantages and disadvantages. Through this review, we sought to identify which of the two agents are better suited to intraoperative brain relaxation. The objective of this review was to compare the effects of mannitol versus those of hypertonic saline on intraoperative brain relaxation in patients undergoing craniotomy.

\section{METHODS}

\section{Criteria for considering studies for this review Types of studies}

We included randomised controlled trials (RCTs) that compared the use of hypertonic saline versus mannitol for brain relaxation. We also included studies in which any other method used for intraoperative brain relaxation was compared with mannitol or hypertonic saline.

We excluded studies in which other methods for producing brain relaxation such as hyperventilation and administration of drugs such as furosemide had not been uniformly used between two study groups. Monitoring of intracranial pressure was not a prerequisite for inclusion of studies in our review.

\section{Types of participants}

We included paediatric and adult participants ( $>18$ years of age) of either gender who received mannitol or hypertonic saline during craniotomy for brain tumour. We excluded neonates (younger than 28 days old) from this review.

\section{Types of interventions}

The experimental intervention was hypertonic saline and the control treatment was mannitol. 


\section{Types of outcome measures}

Primary outcomes

1. Longest follow-up mortality

2. Outcome at 3 months (Glasgow outcome scale score)

3. Adverse events such as electrolyte imbalance, haemodynamic disturbance, rebound oedema and kidney injury.

\section{Secondary outcomes}

1. Brain relaxation (as assessed on three-, four- or five-point scales and reported as dichotomised outcomes: good and poor)

2. Intensive Care Unit (ICU) stay

3. Hospital stay

4. Quality of life assessment.

\section{Search methods for identification of studies} Electronic searches

We searched the Cochrane Central Register of Controlled Trials (2013, Issue 10) [Appendix 1], MEDLINE through OvidSP (1966 to October 2013) [Appendix 2] and EMBASE through OvidSP (1980 to October 2013) [Appendix 3].

The MEDLINE search strategy was combined with the Cochrane highly sensitive search filter for identifying RCTs. ${ }^{[7]}$ The MEDLINE search strategy was adapted for searches of other databases. We applied no language restrictions.

\section{Searching other resources}

We searched for relevant ongoing trials on specific websites such as the following.

1. www.indmed.nic.in

2. www.cochrane-sadcct.org

3. www.clinicaltrials.gov.

Data collection and analysis

\section{Selection of studies}

Using results of the above searches, we screened all titles and abstracts for eligibility. Two review authors (Gyaninder P Singh and Vidhu Anand) independently performed this screening. We obtained and assessed for relevance of the full articles for all the potentially eligible RCTs relevance based on the preplanned checklist. Each review author documented the reason for exclusion of each excluded trial. We resolved disagreements between review authors through discussion with the third review author (Hemanshu Prabhakar), who decided on inclusion or exclusion of the study. We compiled a list of all eligible trials.

\section{Data extraction and management}

Two review authors (Gyaninder P Singh and Vidhu Anand) independently extracted the data and assessed trial quality using a standardised data extraction form. We resolved disagreements through consultation with the third review author (Hemanshu Prabhakar). In cases in which additional information was required, Gyaninder P Singh or Hemanshu Prabhakar contacted the first author of the relevant trial.

\section{Assessment of risk of bias in included studies}

Two review authors independently assessed the methodological quality of the included trials (Vidhu Anand and Gyaninder P Singh). We resolved disagreements through discussion with the third review author (Hemanshu Prabhakar). We performed the assessment as suggested in the Cochrane Handbook for Systematic Reviews of Interventions ${ }^{[8]}$ and judged the risk of bias of included studies on the basis of the following domains.

1. Random sequence generation

2. Allocation concealment

3. Blinding and outcome

4. Incomplete outcome reporting

5. Publication bias and any other bias

6. Follow-up of study participants.

We considered a trial as having low risk of bias if all domains were assessed as adequate. We considered a trial as having high risk of bias if one or more domains were assessed as inadequate or unclear. We included a 'risk of bias' table as part of the characteristics of included studies and a 'risk of bias summary' figure, which detailed all judgements made for all the studies included in the review.

\section{Measures of treatment effect}

We undertook statistical analysis using the statistical software, Review Manager 5.2., ${ }^{[9]}$ of the Cochrane Collaboration. We used risk ratios (RRs) to measure treatment effect for proportions (dichotomous outcomes) among primary outcomes and adverse effects. We converted continuous data to mean differences (MDs) using the inverse variance method and we calculated an overall MD. We used a fixed-effect model when no evidence of significant heterogeneity was found between studies, and a random-effects model when heterogeneity was likely ${ }^{[10]}$ As an estimate of the statistical significance of a difference between experimental and control interventions, we calculated RRs and MDs between groups as well as $95 \%$ confidence intervals (CIs). A statistically significant difference between intervention and control groups was assumed if 95\% CI did not include the value of no differential effect.

Unit of analysis issues

We included in our review only RCTs with a parallel group design. 


\section{Dealing with missing data}

We performed quantitative analysis on an intention-to-treat basis and contacted study authors to obtain missing data. We analysed missing data, if any, by imputation using best- and worst-case scenario methods. If we found insufficient data, the potential impact of the missing data was considered in the interpretation of results.

\section{Assessment of heterogeneity}

We did not perform meta-analysis if we suspected important clinical heterogeneity on examination of the included trials. We used the Q-statistic to test statistical heterogeneity between trials and considered a $P \leq 0.05$ as indicating significant heterogeneity; the $I^{2}$ statistic was used to assess the magnitude of heterogeneity. ${ }^{[11]}$ We considered $I^{2}>50 \%$ to indicate that a meta-analysis was not appropriate and used a random-effects model analysis if $I^{2}$ was between $30 \%$ and $50 \%$. However, the decision to use a random- or fixed-effect model did not rest solely on the value of $I^{2}$ but rather was based on an overall assessment of the heterogeneity of included studies. When in doubt, we carried out both fixed- and random-effects models to examine potential differences.

\section{Assessment of reporting biases}

We assessed publication bias, funding bias and small-study effect in a qualitative manner, using a funnel plot. We planned to test for funnel plot asymmetry if $>10$ studies were included in the meta-analysis.

\section{Data synthesis}

We quantitatively reviewed the included data and combined them by intervention, outcome and population, using Review Manager 5.2. ${ }^{[9]}$ We synthesised data in the absence of important clinical or statistical heterogeneity and expressed RRs for proportions.

\section{Subgroup analysis and investigation of heterogeneity}

When appropriate, given obvious clinical or statistical $\left(I^{2}>40 \%\right)$ heterogeneity, we considered subgroup analysis based on the age of participants (children vs. adults) and on concentrations of hypertonic saline and mannitol. We considered doses of hypertonic saline and mannitol in subgroup analyses if the data indicated heterogeneity on that basis.

\section{Sensitivity analysis}

We performed sensitivity analysis to explore the consistency of effect size measures in trials with low risk of bias versus high risk of bias and to investigate the impact of missing data using the imputation method described above.

\section{Summary of findings}

We planned to use the principles of the Grading of Recommendations, Assessment, Development and
Evaluation (GRADE) system ${ }^{[12]}$ in our review to assess the quality of the body of evidence associated with specific outcomes (mortality, outcome at 3 months, brain relaxation, ICU stay, hospital stay and adverse effects) and to construct a 'summary of findings' (SoF) table using GRADEpro software. The GRADE approach appraises the quality of a body of evidence based on the extent to which one can be confident that an estimate of effect or association reflects the item being assessed. Assessment of the quality of a body of evidence considers within-study risk of bias (methodological quality), directness of the evidence, heterogeneity of the data, precision of effect estimates and risk of publication bias. We created the SoF [Figure 1] for brain relaxation. We found low evidence recommending the use of hypertonic saline for intraoperatOive brain relaxation in patients undergoing surgery for brain tumour; therefore, use of hypertonic saline rather than mannitol is recommended.

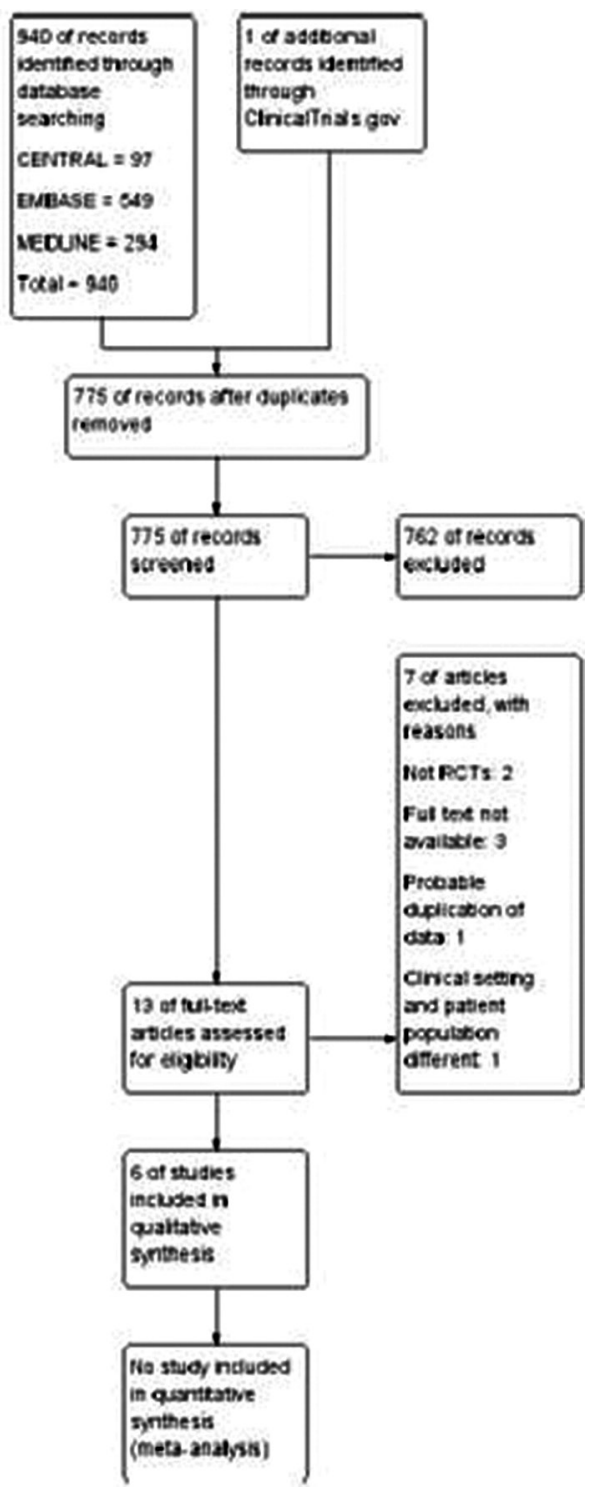

Figure 1: Study flow diagram 


\section{RESULTS}

\section{Description of studies}

Included studies

We included six studies in our review [Figure 1]. ${ }^{[4,5,13-16]}$ All included studies were of parallel design, and only three studies ${ }^{[13-16]}$ used equiosmolar concentrations of fluids. None of the included studies reported our primary outcomes. Brain bulk was reported differently by all of the studies; however, appropriate data were not provided by the authors of three studies. ${ }^{[4,5,14]}$ A single study ${ }^{[16]}$ reported our secondary outcomes of the ICU stay and hospital stay.

\section{Excluded studies}

We excluded seven studies; two studies were not RCTs. ${ }^{[17,18]}$ We were unable to obtain the full text for three studies. ${ }^{[19-21]}$ A probable duplication of data was noted in one study; ${ }^{[22]}$ and in another study, ${ }^{[23]}$ the participant population and the clinical setting were different from those in our inclusion criteria.

\section{Risk of bias in included studies}

We assessed the risk of bias of included studies using the 'risk of bias' tool developed by the Cochrane Collaboration. ${ }^{[11]}$ The risk of bias tool invites judgements on five items for each trial (selection bias, performance bias, detection bias, attrition bias and reporting bias). All review authors independently assessed risk of bias for each study and resolved disagreements by discussion. The characteristics of included studies used for our assessment of the risk of bias in included studies are shown in Figures 2 and 3. Only one study ${ }^{[5]}$ was found to be of high methodological quality.

\section{Allocation (selection bias)}

Of the six included studies, only three ${ }^{[5,14,16]}$ reported allocation concealment. The remaining studies did not describe allocation concealment.

\section{Blinding (performance bias and detection bias)}

Of the six included studies, only three ${ }^{[5,14,16]}$ reported blinding of participants and personnel; four studies

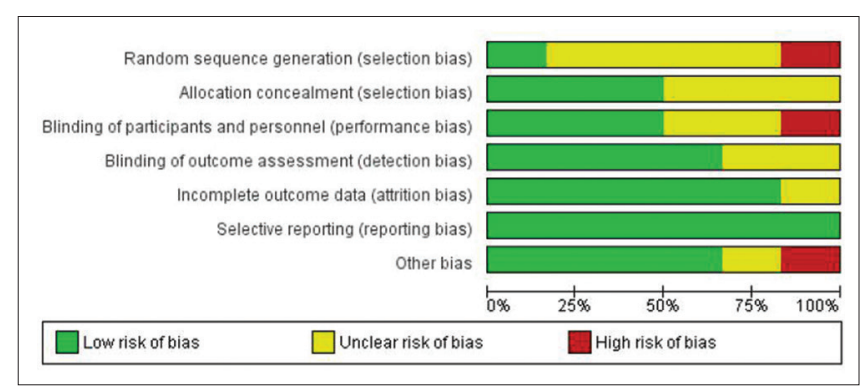

Figure 2: Risk of bias graph: Review authors' judgements about each risk of bias item presented as percentages across all included studies reported blinding of the outcome assessor. ${ }^{[5,14-16]}$ The remaining studies did not describe blinding.

\section{Incomplete outcome data (attrition bias)}

Five studies reported data on all participants. ${ }^{[4,5,14-16]}$ However, this information remained unclear in one study $^{[13]}$ as it was presented as an abstract and study authors failed to include it.

\section{Selective reporting (reporting bias)}

We found that all planned outcomes were reported in the studies. The study authors reported all outcomes mentioned in their methodology.

\section{Other potential sources of bias}

We could find no other potential sources of bias in four of the included studies. ${ }^{[4,5,14,16]}$ In one study, ${ }^{[16]}$ the intervention fluid was donated by a pharmaceutical company and this could have introduced bias into the study. The source of the intervention fluid remained unclear in another study. ${ }^{[13]}$

\section{Effects of interventions Primary outcomes \\ Longest follow-up mortality}

No study reported this outcome.

Outcome at 3 months (Glasgow Outcome Scale score) No study reported this outcome.

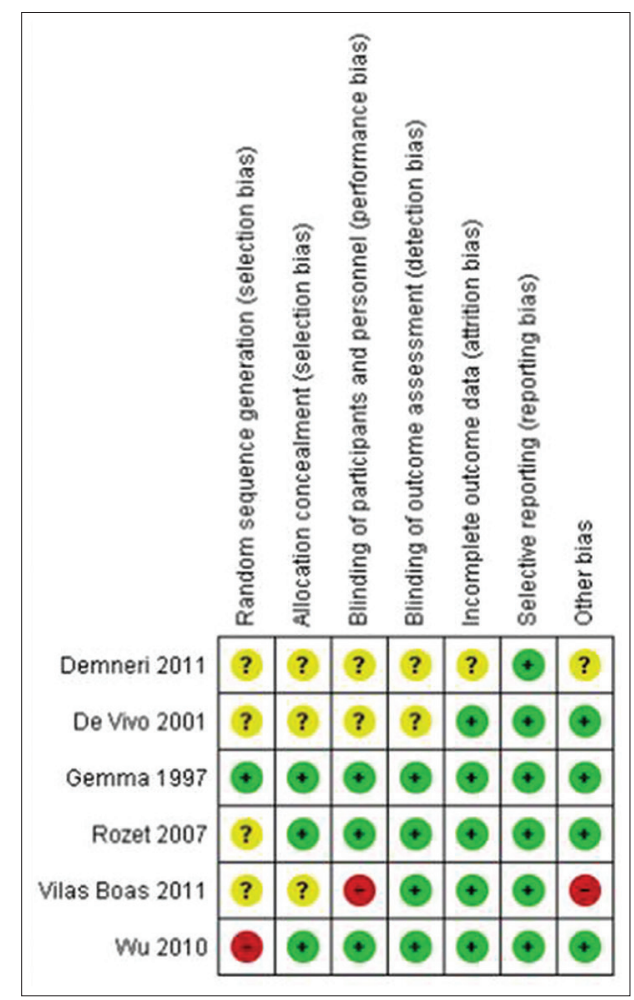

Figure 3: Risk of bias summary: Review authors' judgements about each risk of bias item for each included studies 
Adverse events such as electrolyte imbalance, haemodynamic disturbance, rebound oedema and kidney injury

No study reported these outcomes.

None of the studies reported our primary outcomes of longest follow-up mortality, Glasgow outcome scale score at 3 months and adverse events such as electrolyte imbalance, haemodynamic disturbance, rebound oedema and kidney injury.

\section{Secondary outcomes}

\section{Brain relaxation}

Three studies enrolling 387 participants reported brain relaxation $(73.4 \%$ of total participants in this review). ${ }^{[13,15,16]}$ These three trials suggest that the incidence of inadequate brain relaxation was reduced from 68 of 190 in the mannitol group to 42 of 197 in the hypertonic saline group (RR of brain bulge 0.60, 95\% CI - 0.44-0.83, $P-0.002)$. No heterogeneity was noted in these studies [Figure 4].

\section{Intensive Care Unit and hospital stay}

Only one study ${ }^{[16]}$ enrolling 238 participants reported the ICU stay and hospital stay (45.2\% of total participants in this review). This study suggested that the mean (standard deviation [SD]) duration of ICU stay in the mannitol and hypertonic saline groups was $1.28(0.5)$ and $1.25(0.5)$ days $(P-0.64)$, respectively; the mean (SD) duration of hospital stay in the mannitol and hypertonic saline groups was $5.7(0.7)$ and $5.7(0.8)$ days $(P-1.00)$, respectively.

\section{Quality of life assessment}

No study reported this outcome.

\section{DISCUSSION}

This review concerns randomised evidence for the use of hypertonic saline and mannitol in patients undergoing surgery for brain tumour. We planned to collect data on clinically relevant outcomes such as mortality, outcome at 3 months and adverse events (primary), along with other parameters (secondary outcomes) such as intraoperative brain relaxation, length of ICU and hospital stay and quality of life. Data on the primary end points of our review were lacking. However, we were able to collect data for the incidence of intraoperative brain relaxation in study participants receiving two fluids.

None of the studies reported our primary outcomes. Only three studies reported our secondary outcomes. Our analysis suggests that hypertonic saline is beneficial in producing brain relaxation in patients undergoing surgery for brain tumour. Length of ICU stay and length of hospital stay were comparable after intraoperative use of hypertonic saline or mannitol.

The overall methodological quality of these studies cannot be considered good, but no heterogeneity was noted. However, this evidence was obtained from a limited number of studies. We were unable to retrieve data on many clinically useful outcomes such as mortality, outcome at 3 months and quality of life. The evidence produced by this review, therefore, should be interpreted with caution, keeping in mind that it is only intraoperative brain relaxation that may be achieved more effectively with the use of hypertonic saline.

We selected randomised studies for our review, and most of these studies did not report details of randomisation and allocation concealment. However, blinding was carried out in most. The overall methodological quality of these studies could not be considered good. The included studies had homogeneous populations, and no heterogeneity was noted. For brain relaxation, the quality of evidence was low as suggested by the SoF [Table 1].

In an attempt to minimise bias, we followed the guidelines provided in the Cochrane Handbook for

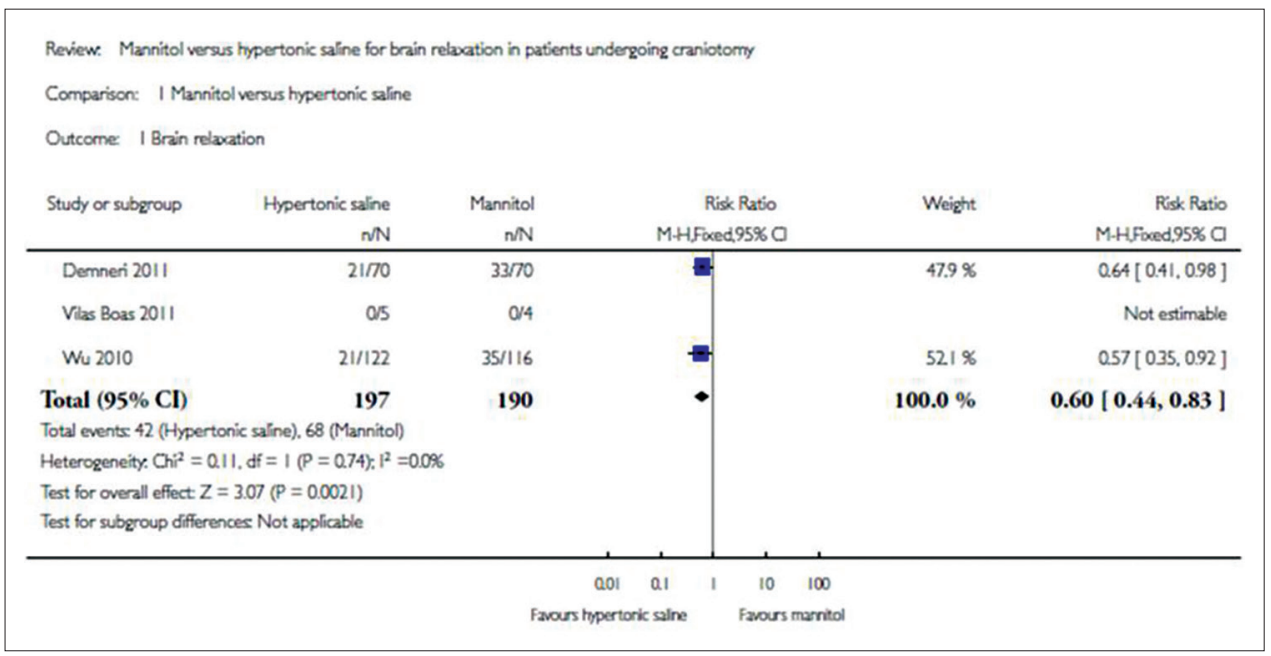

Figure 4: Forest plot of comparison mannitol versus hypertonic saline for outcome: Brain relaxation 


\section{REFERENCES}

1. Battison C, Andrews PJ, Graham C, Petty T. Randomized, controlled trial on the effect of a $20 \%$ mannitol solution and a $7.5 \%$ saline $/ 6 \%$ dextran solution on increased intracranial pressure after brain injury. Crit Care Med 2005;33:196-202.

2. Schwarz S, Georgiadis D, Aschoff A, Schwab S. Effects of hypertonic $(10 \%)$ saline in patients with raised intracranial pressure after stroke. Stroke 2002;33:136-40.

3. Vialet R, Albanèse J, Thomachot L, Antonini F, Bourgouin A, Alliez B, et al. Isovolume hypertonic solutes (sodium chloride or mannitol) in the treatment of refractory posttraumatic intracranial hypertension: $2 \mathrm{~mL} / \mathrm{kg} 7.5 \%$ saline is more effective than $2 \mathrm{~mL} / \mathrm{kg}$ 20\% mannitol. Crit Care Med 2003;31:1683-7.

4. De Vivo P, Del Gaudio A, Ciritella P, Puopolo M, Chiarotti F, Mastronardi E. Hypertonic saline solution: A safe alternative to mannitol $18 \%$ in neurosurgery. Minerva Anestesiol 2001;67:603-11.

5. Gemma M, Cozzi S, Tommasino C, Mungo M, Calvi MR, Cipriani A, et al. $7.5 \%$ hypertonic saline versus $20 \%$ mannitol during elective neurosurgical supratentorial procedures. J Neurosurg Anesthesiol 1997;9:329-34.

6. White H, Cook D, Venkatesh B. The use of hypertonic saline for treating intracranial hypertension after traumatic brain injury. Anesth Analg 2006;102:1836-46.

7. Lefebvre C, Manheimer E, Glanville J. Searching for studies. In: Higgins JP, Green S, editors. Cochrane Handbook for Systematic Reviews of Interventions Version 5.1.0. Ch. 6. The Cochrane Collaboration; 2011. Available from: http:// www.cochrane-handbook.org. [Last updated on 2011 Mar].

8. Higgins JP, Altman DG, Sterne JA. Assessing risk of bias in included studies. In: Higgins JPT, Green S, editors. Cochrane Handbook for Systematic Reviews of Interventions Version 5.1.0. Ch. 8. The Cochrane Collaboration; 2011. Available from: http://www.cochrane-handbook. org. [Last updated on $2011 \mathrm{Mar}$.

9. Review Manager (RevMan) 5.2. Copenhagen [Computer Program]. Version 5.2. Copenhagen: The Nordic Cochrane Centre, The Cochrane Collaboration; 2013.

10. DerSimonian R, Laird N. Meta-analysis in clinical trials. Control Clin Trials 1986;7:177-88.

11. Higgins JP, Thompson SG. Quantifying heterogeneity in a meta-analysis. Stat Med 2002;21:1539-58.

12. Guyatt GH, Oxman AD, Kunz R, Vist GE, Falck-Ytter Y, Schünemann HJ; GRADE Working Group. What is "quality of evidence" and why is it important to clinicians? BMJ 2008;336:995-8.

13. Demneri M, Hoxha A, Pilika K, Saraci M. Comparison of $20 \%$ mannitol and $7.5 \%$ hypertonic saline for supratentorial craniotomy. Eur J Anaesthesiol 2011;28:106-7.

14. Rozet I, Tontisirin N, Muangman S, Vavilala MS, Souter MJ, Lee LA, et al. Effect of equiosmolar solutions of mannitol versus hypertonic saline on intraoperative brain relaxation and electrolyte balance. Anesthesiology 2007;107:697-704.

15. Vilas Boas WW, Marques MB, Alves A. Hydroelectrolytic balance and cerebral relaxation with hypertonic isoncotic saline versus mannitol $(20 \%)$ during elective neuroanesthesia. Rev Bras Anestesiol 2011;61:456-68.

16. Wu CT, Chen LC, Kuo CP, Ju DT, Borel CO, Cherng CH, et al. A comparison of 3\% hypertonic saline and mannitol for brain relaxation during elective supratentorial brain tumor surgery. Anesth Analg 2010;110:903-7.

17. Levin AB, Duff TA, Javid MJ. Treatment of increased intracranial pressure: A comparison of different hyperosmotic agents and the use of thiopental. Neurosurgery 1979;5:570-5.

18. Smedema RJ, Gaab MR, Heissler HE. A comparison study between mannitol and glycerol therapy in reducing intracranial pressure. Intracranial Pressure 1993;VIII: 605-8.

19. Eldahab HA, Awad W, Wagh O. Should hypertonic saline 3\% replace mannitol $20 \%$ for reduction of intracranial pressure in craniotomy for supratentorial tumors? A comparative study. Egypt J Anaesth 2009;25:413-28.

20. Erard A, Bracco D, Walder B, Ravussin P. Effect of mannitol, $\mathrm{NaCl} 7.5 \%$ and $\mathrm{NaCl} 0.9 \%$ on osmolality and osmolarity: A randomized controlled study in neurosurgical patients [abstract]. Br J Anaesth 1999;82 Suppl 1;87(A287).

21. Pausawasdi S, Bunyaratavej S. Furosemide and mannitol - Induced changes in intracranial pressure during the removal of intracranial tumours. J Med Assoc Thai 1982;65:413-9.

22. Muangman S, Rozet I, Vavilala MS, Lee LA, Lam AM. Iso-osmolar solutions of mannitol vs. hypertonic saline for intraoperative brain relaxation. Anesthesiology 2005;103:A76.

23. Harutjunyan L, Holz C, Rieger A, Menzel M, Grond S, Soukup J. Efficiency of $7.2 \%$ hypertonic saline hydroxyethyl starch 200/0.5 versus mannitol 15\% in the treatment of increased intracranial pressure in neurosurgical patients - a randomized clinical trial [ISRCTN62699180]. Crit Care 2005;9:R530-40.

\section{APPENDICES}

\section{Appendix 1: Cochrane Central Register of Controlled Trials search strategy}

\#1 MeSH descriptor Mannitol explode all trees

\#2 mannitol*

\#3 (\#1 OR \#2)

\#4 MeSH descriptor Craniotomy explode all trees

\#5 MeSH descriptor Neurosurgical Procedures explode all trees

\#6 MeSH descriptor Neurosurgery explode all trees

\#7 MeSH descriptor Intracranial Pressure explode all trees

\#8 MeSH descriptor Intraoperative Period explode all trees

\#9 (brain near (surg* or manipulat* or procedur* or relax*)) or craniotom* or (neurosurg* near (patient* or procedur*

or manipulat*)) or (intracranial near pressure)

\#10 (\#4 OR \#5 OR \#6 OR \#7 OR \#8 OR \#9)

\#11 (\#3 AND \#10) 


\section{Appendix 2: MEDLINE (Ovid SP) search strategy}

1. exp Mannitol/or mannitol*.af.

2. exp Craniotomy/or Neurosurgical Procedures/or Neurosurgery/or Intracranial Pressure/or Intraoperative Period/or (brain adj3 (surg* or manipulat* or procedur* or relax*)).mp. or craniotom*.af. or (neurosurg* adj3 (patient* ${ }^{*}$ or procedur ${ }^{*}$ or manipulat $\left.{ }^{\star}\right)$ ).mp. or (intracranial adj3 pressure).mp.

3. 1 and 2

4. ((randomised controlled trial or controlled clinical trial).pt. or randomised.ab. or placebo.ab. or drug therapy.fs. or randomly.ab. or trial.ab. or groups.ab.) not (animals not (humans and animals)).sh.

5. 3 and 4

\section{Appendix 3: EMBASE (Ovid SP) search strategy}

1. exp mannitol/or mannitol*.af.

2. $\exp$ craniotomy/or neurosurgery/or intracranial pressure/or intraoperative period/or (brain adj3 (surg* or manipulat* or procedur* or relax*)).mp. or craniotom*.af. or (neurosurg* adj3 (patient* or procedur* or manipulat*)).mp. or (intracranial adj3 pressure).mp.

3. (randomised-controlled-trial/or randomisation/or controlled-study/or multicenter-study/or phase-3-clinical-trial/or phase-4-clinical-trial/or double-blind-procedure/or single-blind-procedure/or (random* ${ }^{*}$ cross?over* or multicenter* ${ }^{*}$ factorial ${ }^{*}$ or placebo* $^{*}$ or volunteer*).mp. or ((singl ${ }^{*}$ or doubl ${ }^{*}$ or trebl* $^{*}$ or tripl ${ }^{*}$ ) adj3 (blind* or mask*)).ti, ab. or (latin adj square).mp.) not (animals not (humans and animals)). sh.

4. 1 and 2 and 3 